\title{
Natural and Large Catastrophes - Changing Risk Characteristics and Challenges for the Insurance Industry
}

\author{
John R. Coomber
}

SwissRe, Mythenquai 50/60, PO Box CH-8022, Zurich, Switzerland.

E-mail: Silvia_Kauer@swissre.com

The insurance industry is confronted with ever bigger losses due to large disasters as well as with a series of new risks whose risk profile is extremely difficult to quantify. This paper deals with the emergence of these new risks and the challenges that the industry will face in managing them.

The Geneva Papers (2006) 31, 88-95. doi:10.1057/palgrave.gpp.2510067

Keywords: risk management; emerging risks; natural catastrophes; nanotechnology

\section{Introduction}

At USD 49 billion, 2004 saw new records in worldwide insured catastrophe losses. The December tsunami, multiple hurricanes striking the U.S., the terrorist bomb attack in Madrid - in recent years both the number of victims and the cost of damages have trended higher to their now unprecedented levels (Figures 1 and 2). ${ }^{1}$ This progression may be largely explained by an increased concentration of population and property values in historically exposed geographies. Nevertheless, this trend raises important questions for the insurance industry.

How will the size and correlation of existing risks develop? What new risks will emerge? What consequences does this imply for the insurance industry?

\section{Changing risk characteristics}

The scope and inter-connectedness of the global risk landscape is growing. The size of potential losses increases, while loss correlations are compounded by advances in technology and the expansion of networks, both virtual and physical. Power supply grids, for example, become increasingly widespread and interconnected, while economies and societies become more dependent upon their uninterrupted service. Familiar risks such as power failures are leading to unforeseen claimants such as tenants and employees, in addition to the direct customers of the electric utilities. Today's increased mobility allows consequences to proliferate faster, over greater distances and on a wider scale. Familiar hazards such as the influenza virus have a

\footnotetext{
${ }^{1}$ Swiss Re (2005). Catastrophes captured by the Sigma database are defined by thresholds in terms of insured losses (property and business interruption) or number of victims.
} 


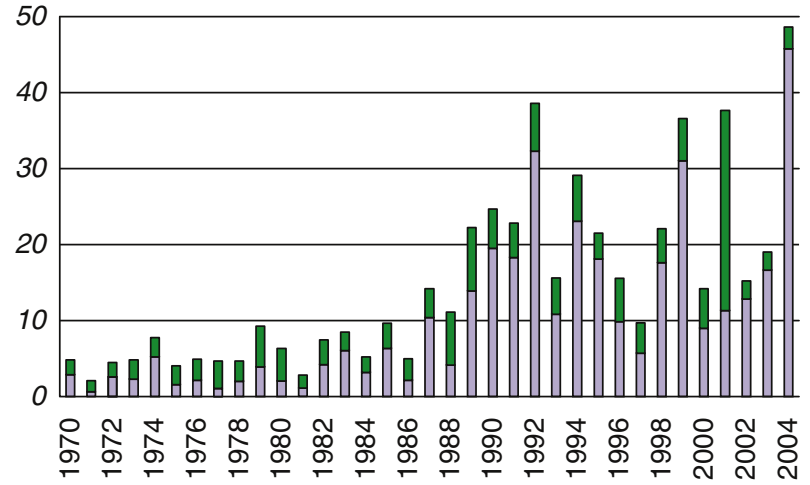

$\square$ Natural catastrophes $\quad$ Man-made disasters

Figure 1. Insured catastrophic losses 1970-2004 (market losses in USD bn indexed to 2004 values). Source: Swiss Re (2005). Catastrophes captured by the Sigma database are defined by thresholds in terms of insured losses (property and business interruption) or number of victims.

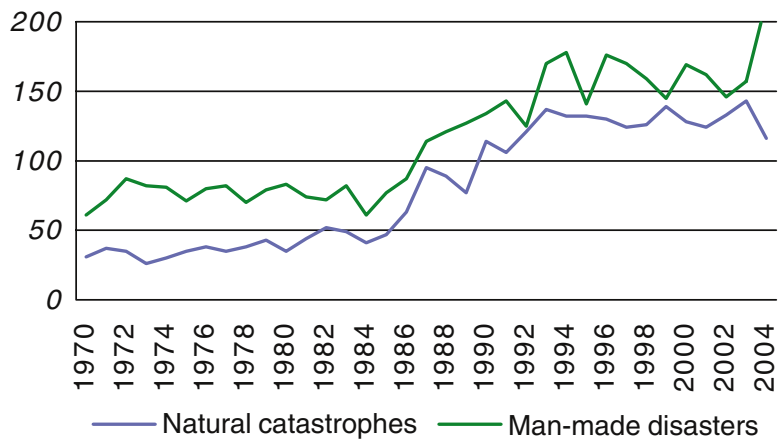

Figure 2. Number of events 1970-2004. Source: Swiss Re (2005). Catastrophes captured by the Sigma database are defined by thresholds in terms of insured losses (property and business interruption) or number of victims.

distressing potential for fatalities and economic disruption spanning geographies, spreading over larger time periods and affecting multiple lines of insured business.

Advances in technology and the speed of their evolution into commercialised products challenge the building of loss histories and the deciphering of interdependencies. Simple cause-and-effect relationships are steadily replaced by multi-causal and multi-conditional systems, ultimately complicating questions of responsibility, culpability and liability. While society naturally tries to reduce the probability of disasters, the cost of individual occurrences may escalate. For example, state-of-the-art rail transport technologies reduce the chances of an accident; however, increasing train speeds geometrically increase an accident's effects.

Additional uncertainty is generated by shifts in society's perception of value. The concept of loss is ultimately connected to the understanding of value. Hence, the future 
risk landscape can also change as a consequence of varying perceptions as to what constitutes appropriate compensation for a loss event.

Apart from addressing the measurement of this risk evolution, the insurance industry has to improve clarity as to the scope of coverage. Wordings are not always clear in differentiating covered risks determined, for example, in event definitions, losses triggered by failures in operational risk or emerging from commercial misjudgements.

\section{The limits of insurability}

Growing exposures and concentrations drive up insurers' capital requirements and challenge the balance sheet in respect to both profitability and insurability. In an increasingly interconnected environment, correlations among independently assessable risks become less clear. Some risk factors - for example, property risks, market risks and credit risks - may behave independently under "normal" circumstances, but exhibit correlation in extreme scenarios, so-called tail correlation. Seemingly innocuous trends such as yearly changes in global temperatures may, if persistent, have dramatic, even uninsurable, effects over time. Three risk categories are examined in more detail below.

\section{Natural catastrophes}

Twenty-five years ago, the modelling of natural catastrophes was in its infancy; today, assessment of these risks is approached with confidence. Mother Nature is still an unpredictable business partner; however, knowledge of extreme events has improved dramatically over the past few decades. This understanding has flowed into hazard maps, construction standards, pricing models and emergency planning programmes, and, consequently, a steadily increasing demand for insurance against natural catastrophes has been met by a rising willingness of insurers to cover such risks.

Nevertheless, natural hazards are still a key concern due to their cumulative risk properties and the huge capacity that must be allocated to cover them. In spite of major annual fluctuations, a trend emerges over the past 30 years showing a dramatic rise in natural catastrophe insurance losses. This increase is principally a result of rising population densities in general and greater insurance penetration in high-risk areas. Given that these trends have been consistent for decades, natural hazard losses will likely continue to rise.

Furthermore, a growing body of scientific research supports the view that the frequency and intensity of certain natural catastrophes can be expected to rise beyond the normal cyclical fluctuations. Temperature measurements indicate that, overall, the earth's lower atmosphere has warmed over the past hundred years. Due to the physical characteristics of the atmosphere, it is highly probable that a global temperature increase will lead to an intensification of the hydrological cycle. Global climate models predict increased and more frequent seasonal precipitation in various regions of the world. It is feared that this might lead to more frequent and/or extreme flood events and exacerbate storm activity in general (Figure 3). Additionally, life and health 


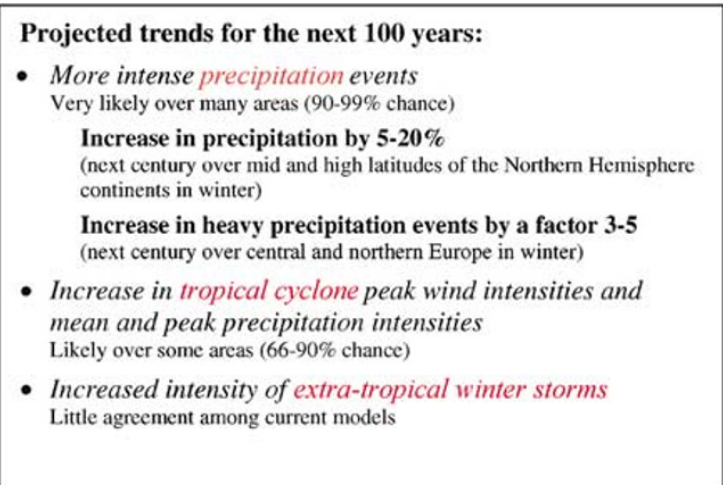

Figure 3. Projected trends for the next 100 years. Source: IPCC (2001) and OcCC (2003).

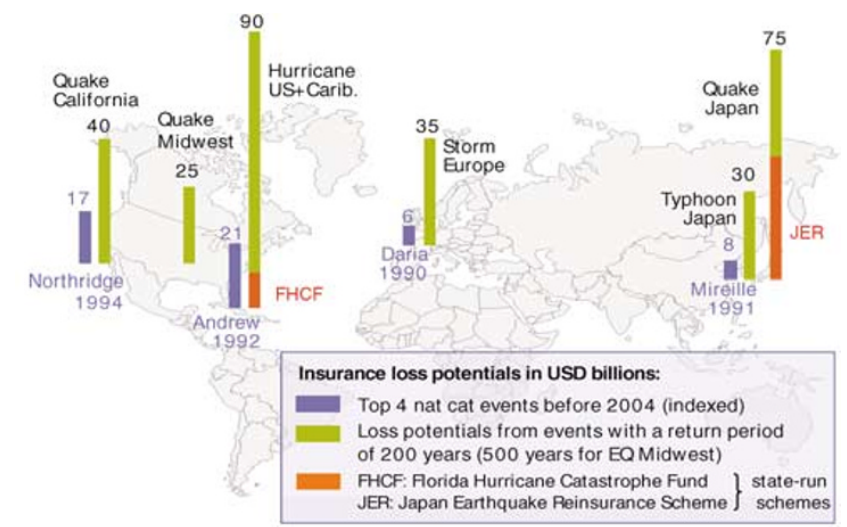

Figure 4. Insurance loss potentials in USD billions. Source: Swiss Re Nat Cat R\&D.

insurance may also be affected, for example, as global warming and changing climatic zones encourage the spread of vector-bourne diseases.

Greater exposures require large amounts of capital as peak risks do not diversify. As the cost of servicing that capital is high, the industry is capturing new ways of expanding capacity. One such option is the securitisation of risks through the issuance of catastrophe bonds. The capital markets to date have borne only a small fraction of such large-scale risks. At the end of 2004, the total market value of outstanding insurance-linked securities (ILS) for natural catastrophes was USD 4.4 billion. ${ }^{2}$ This is still a very small amount relative to the loss potential involved (Figure 4).

Traditional insurance is positioned to work with the trends it sees in the area of natural catastrophe. Climate change should continue to be monitored, attempts made to decipher the potential impact, and "sustainability agendas" promoted accordingly.

${ }^{2}$ Includes "Vita Capital" life catastrophe bond. 
The industry should continue to exploit technological and scientific advances to improve its modelling capability, and also develop the potential of new risk transfer solutions. Fresh approaches to tapping the financial markets and sharing risk with the public and governments will likely emerge, either from the insurance industry or elsewhere.

\section{Terrorism risk}

Prior to 11 September 2001, terrorism risk loss potentials appeared manageable for the private insurance industry. Appearances have changed. The industry has been compelled to fundamentally review its risk acceptance position, while awareness of and demand for protection against this threat has surged. This has resulted in changes in the assessment and pricing of terrorism coverage, as well as the impositions of reductions and limits to prevent unacceptable exposures.

Terrorism is difficult to define in unambiguous terms, as it cannot be readily distinguished from all other forms of violence, including war. As with any risk however, the insurance industry, policyholders and public stakeholders have to agree on a common understanding and definition of the loss event. The sheer size of the potential scenarios and the difficulty in assessing their probability are another barrier. In the immediate aftermath of 11 September, insurers found international terrorism to be basically uninsurable, and responded by limiting cover and charging an additional premium. Improved approaches to assess and control exposures and to price covers began evolving immediately.

In some ways, terrorism risk parallels natural catastrophe risk. In both cases, enormous loss potentials make diversification difficult to achieve; individual events can affect entire economies and many different lines of business. The accumulation potential among the different lines of business is much higher than previously assumed - not only for terrorism but also for other man-made exposures - and must be better controlled. However, there is also a significant difference: the probabilities and consequences of natural hazards can be simulated with scientific methods and models. This is far more difficult for terrorism risk, which tests the principles of insurability (Figure 5).

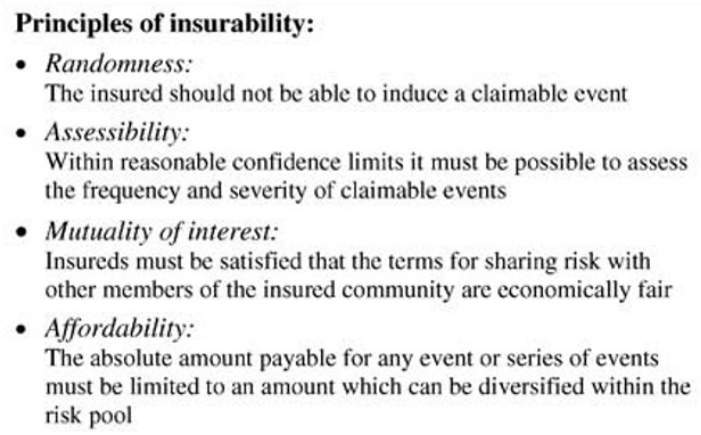

Figure 5. Principles of insurability. 
To overcome these difficulties, various measures were and are being taken to make terrorism manageable. These include:

- coordinating insurance capacity in individual markets with existing private and state-run schemes;

- improving exposure monitoring with more detailed information;

- imposing high organisational, technical and personal safeguarding requirements on objects which are particularly at risk;

- limiting capacity for individual major risks.

Several state-sponsored programmes for risk pooling were established following 11 September (e.g. TRIA in the U.S., GAREAT in France and Extremus VersicherungsAG in Germany), complementing the few national schemes already in place prior to the event (e.g. Pool Re in the U.K.). Some major hurdles to insurability can be overcome through such state involvement. As the insurer of last resort, the state is in a better position to deal with extreme loss potentials than private insurers with their limited capital and capacity. Also, governments can declare terrorism insurance mandatory, for example, through inclusion in property policies against an additional premium. This step would greatly expand the risk community, spreading the risk throughout an entire society.

\section{Nanotechnology}

Nanotechnology refers to particles, coatings or materials smaller than 100 nanometre $(\mathrm{nm})$, with specific properties enabling innovative uses in a broad range of products. Some studies indicate, however, that nano-particles may also cause adverse health effects due to their particular characteristics. Indeed, their size makes them highly mobile in both the environment and the human body. Nano-particles appear to be capable of crossing the blood-brain barrier with uncertain consequences to human health.

Nanotechnology is not a manageable or even yet definable field of risk. It is on the insurance industry's agenda because of concerns over "nano-toxicity" or "nanopollution" and because nano-products are expected to become omnipresent across industry sectors and countries. This introduces the possibility of long-latent, widespread and unforeseen claims. Unnecessary delays in establishing standards and regulations to treat the risk potential need to be avoided.

Four issues confront the insurability of "nano-risks". First, scientific evaluations of potential health and environment risks are few and remain inconclusive. Second, there are no regulatory guidelines that address potential risks in an adequate manner. Furthermore, there is no global approach towards finding a solution satisfactory to business, scientists and regulators alike. Third, there is no common terminology for the great variety of nano-substances, products and applications. Any structured approach towards evaluating potential risk requires a standardisation of materials and processes and a common language for comparison of scientific knowledge. Last but not least, there is no such thing as the nanotechnology industry. Many companies use nanotechnology as one component of their products. However, only a few - primarily 
Expected nanotechnology revenues by 2015

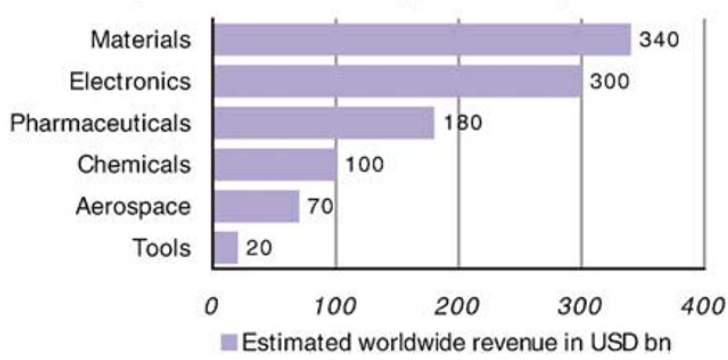

Figure 6. Expected nanotechnology revenues by 2015. Source: Dr. M.C. Roco, National Science Foundation, "The Future of the National Nanotechnology Initiative", 2003.

small start-up companies - deal with nanotechnology exclusively. The diverse multitude of players involving almost all industry segments cannot be dealt with in one and the same way (Figure 6).

At a more philosophical level, it remains to be seen whether nanotechnology exhibits an evolutionary development, like many previous innovative technologies, or becomes revolutionary. The potential to assess the scale, location and time of occurrence of nanotechnology-caused damages is still a widely open question.

Going forward, these uncertainties regarding potential losses need to be reduced. The legal framework and current level of scientific understanding of this risk do not yet permit reliable judgements. Preconditions for achieving this goal are internationally accepted standards and norms (i.e. a common language on how to define and name nano-particles and materials), exposure assessments (who is in contact with a given material and to what extent; what are the health consequences, if any) and a legal framework (e.g. approval processes, disclosure obligations). At present, the key to best practice in underwriting includes building up specific knowledge enabling the analysis of products and applications on a case-by-case basis. The ongoing dialogue and co-operation with all stakeholders needs full attention, while public perception is continuously monitored. Combined efforts need to be made to either prove or refute the harmlessness of nanotechnology in order to find ways to apply the technology without being hit by any unwelcome surprises.

\section{Consequences and questions for the insurance industry}

The insurance industry provides an indispensable service to society by pricing and diversifying risk and by partially absorbing the impact of inevitable shocks through its capital base. The industry's ability to respond to large events is critical to performing this service. In a world where risk exposures grow more rapidly than the capital base of insurers and where there is an increasing level of connectivity such that large events provoke secondary risk scenarios, the industry cannot only rely on the solutions of the past. The following issues suggest some of the topics that will occupy the agenda of management going forward. 
- Tail correlations and the limitations of diversification can be better measured and solutions designed accordingly.

- Improved modelling of current catastrophic scenarios such as lethal epidemic, liability and terrorism, as well as extreme natural catastrophes such as tsunamis or volcanic eruptions.

- Partnership through honest dialogue with governmental organisations regarding the size limitations of commercial insurance and the response to any gap.

- Greater transparency of risk via engaged dialogue with academia, industry and public entities on new risks, such as climate change and new technologies.

- Imagination to anticipate, plan for, cover and help mitigate extreme events.

- Explore ways to contribute to uninsured communities; support governments in creating programmes that enable insurability and securitisation.

- Manage post-event situations effectively, being recognised for delivering on promises at times of distress.

\section{References}

Roco, M.C. (2003) The Future of the National Nanotechnology Initiative, Arlington, VA: National Science Foundation.

Swiss Re (2005) 'Natural catastrophes and man-made disasters in 2004', Sigma 1: 1-36.

\section{About the Author}

John R. Coomber was appointed Chief Executive Officer of Swiss Re Group on 1 January 2003. He became a member of the Executive Board in April 1995, responsible for the Group's Life \& Health division. In June 2000, he became a member of the Executive Board Committee. He will retire at the end of 2005. 